\title{
Results From VLA Observations of Five Spiral Galaxies In The Virgo Cluster Region $\dagger$
}

\author{
M. C. Toribio and J. M. Solanes \\ Institut de Ciències del Cosmos - Department d'Astronomia i Meteorologia, \\ Universitat de Barcelona, c/Martí i Franquès 1, E-08028, Barcelona, Spain \\ email: mctoribio@am.ub.es,jm.solanes@ub.edu
}

\begin{abstract}
New HI synthesis observations have been made for the five spiral galaxies NGC 4307, NGC 4356, NGC 4411a/b and NGC 4492, located in the Virgo Cluster Region, with the Very Large Array in its CS configuration. All these objects, but NGC 4411a, belong to a sample of spirals identified in previous single-dish studies of the HI distribution within the Virgo Cluster and its outskirts. The thirteen members of that sample were selected for showing neutral gas deficiencies as strong as those of the inner galaxies of Virgo and other rich clusters, but apparently lying on the infall region.

The goal of the present observations is to progress further on the identification of the external mechanisms that may be responsible for the reduction of the interstellar gas in spiral galaxies and thus contribute to the study of the evolution of galaxies in high-density environments. In particular, the results of this work are relevant for the ongoing debate on the pre-processing of galaxies prior to entering the cluster environment.
\end{abstract}

Keywords. galaxies: spiral, galaxies: evolution, galaxies: ISM, galaxies: clusters: individual

\section{Main Results}

After the data reduction, which ended up with the non-detection of NGC 4492, our analysis of the first moments of the $\mathrm{H}$ I distribution for each of the target galaxies confirms that two of them, NGC 4307 and NGC 4356, are strong gas deficient spirals. Therefore, any attempt to measure the rotational velocity of these galaxies from their reduced H Idisks will possibly underestimate its real value. This has to be taken into account when deriving the radial distance from the Tully-Fisher relation. As for NGC 4411b, we find a lesser H I-deficiency than previous estimates, whereas NGC 4411a shows a mild gas deficiency. We do not find obvious signs of ongoing interactions with neighbours or tidal features (e.g. gas tails) that could explain the lack of atomic gas in any of these objects.

Furthermore, we have also reviewed minutely all the information available in the literature from previous determinations of the Tully-Fisher distances to these spiral galaxies, finding that the uncertainties in their intrinsic luminosity are dominated by systematic differences in the adopted templates of the Tully-Fisher relation. However, the most significant disagreements on their radial distances come from the values of the rotation velocity adopted by the different authors. These differences arise mainly from the use of H I data catalogues compiled from heterogeneous sources and in which the quoted measurements for our target galaxies do not always agree with the original value. Although this situation cannot necessarily be generalized to the rest of the Virgo galaxies, we suggest a profound revision of the $\mathrm{H}$ I data in order to achieve a more precise characterization of the three-dimensional structure of this region of the sky.

$\dagger$ We thank Juan M. Uson for his help with the preparation of the observe files and his guidance during the data reduction process. 